\title{
Effects of radical cystectomy, radiotherapy, and chemotherapy on the risk of long-term heart-specific death in bladder cancer patients
}

\author{
Fuhan Yang ${ }^{1,2 \#}$, Cheng $\mathrm{Li}^{1,2 \#}$, Yadong Guo ${ }^{1,2 \#}$, Yang $\mathrm{Yu}^{1,2}$, Shiyu Mao ${ }^{1,2}$, Ruiliang Wang ${ }^{1,2}$, Wentao Zhang ${ }^{1,2}$, \\ Aihong Zhang ${ }^{3}$, Xudong Yao ${ }^{1,2}$ \\ ${ }^{1}$ Department of Urology, Shanghai Tenth People's Hospital, School of Medicine, Tongji University, Shanghai, China; ${ }^{2}$ Institute of Urologic \\ Oncology, School of Medicine, Tongji University, Shanghai, China; ${ }^{3}$ Department of Medical Statistics, Tongji University School of Medicine, \\ Shanghai, China \\ Contributions: (I) Conception and design: F Yang, Y Guo; (II) Administrative support: X Yao; (III) Provision of study materials or patients: C Li, \\ S Mao; (IV) Collection and assembly of data: Y Yu, R Wang, W Zhang; (V) Data analysis and interpretation: F Yang, A Zhang; (VI) Manuscript \\ writing: All authors; (VII) Final approval of manuscript: All authors. \\ \#These authors contributed equally to this work. \\ Correspondence to: Prof. Aihong Zhang. Department of Medical Statistics, School of Medicine, Tongji University, Shanghai 200092, China. \\ Email: zhangah@tongji.edu.cn; Prof. Xudong Yao. Department of Urology, Shanghai Tenth People’s Hospital, School of Medicine, Tongji University, \\ Shanghai, China. Email: yaoxudong1967@163.com.
}

Background: At present, the low risk of bladder cancer (BCa)-specific death has allowed for investigation into treatment-related cardiotoxicity. To aid clinicians in selecting appropriate cardiovascular disease screening strategies and interventions, this study explored the heart-specific mortality and prognostic factors of patients with $\mathrm{BCa}$ after radical cystectomy $(\mathrm{RC})$, radiotherapy $(\mathrm{RT})$, or chemotherapy (CT), and compared their long-term heart-specific mortality with that of the general male population.

Methods: We identified three different treatments for BCa patients from the Surveillance, Epidemiology, and End Results (SEER) database: RC, RT, and CT. Patients were included from 2000 to 2012 and followed through 2015. A cumulative mortality curve and competitive risk regression model were applied to evaluate the prognostic factors of heart-specific mortality, and standardized mortality ratios (SMRs) were calculated.

Results: Of 39,500 men, 30.3\%, 18.8\%, and 50.9\% received RC, RT, and CT, respectively. For patients with a survival period of less than 50 months, tumor-specific death exhibited a rapidly increasing trend, which subsequently flatlined. However, the rates heart-specific mortality and other causes exhibited a tendency to increase stably. The heart-specific and all-cause mortality rates of patients in any age group treated with the three abovementioned strategies were higher than those of the general population. The heart-specific mortality of patients with carcinoma in situ treated with RC and CT exceeded their all-cause mortality, while that of other tumor stages did not. The risks of heart-specific [sub-distribution hazard ratio $(\mathrm{SHR})=1.38$; 95\% confidence interval (CI): 1.22-1.57] and tumor-specific (SHR =1.68; 95\% CI: 1.60-1.77) deaths in patients who received RT were higher than those of patients who underwent CT.

Conclusions: The risks of heart-specific and tumor-specific deaths in patients who received RT were higher than those of the RC and CT groups, especially in patients over 65 years of age who received RT.

Keywords: Bladder cancer (BCa); cardiovascular disease; competing risk; Surveillance, Epidemiology, and End Results (SEER)

Submitted Aug 26, 2021. Accepted for publication Oct 22, 2021.

doi: $10.21037 /$ tau-21-835

View this article at: https://dx.doi.org/10.21037/tau-21-835 


\section{Introduction}

Bladder cancer (BCa) is the most common malignant tumor of the urinary system, and its incidence increasing year by year with the growing and ageing population more likely affects older people and men (1,2). Between 1990 and 2016, the number of deaths from BCa increased by 1.6 times $\{113,414$ [95\% confidence interval (CI): 110,361$117,066]$ to 186,199$\}$ (3). Many factors are known to be risk factors for $\mathrm{BCa}$, such as exposure to environmental and occupational chemicals and tobacco smoke, but when these risk factors are removed, the mortality rate of $\mathrm{BCa}$ patients does not decrease significantly (4-6).

Until recently, BCa treatment had seen little progress. For 30 years, clinicians offered the same, limited range of therapeutics to patients, and the 5-year survival rates were flat (7-9). Further analysis of the causes of death in patients with BCa showed that tumor-specific deaths account for a large proportion, but the proportion of non-tumor-specific deaths is also surprisingly high, especially heart-specific mortality (10). Heart-specific deaths were defined as those with an underlying cause of death in the ICD category of diseases of the heart (e.g., coronary heart disease, heart failure, atrial fibrillation, and myocardial infarction) because this category has been the most inclusive and has had the most consistent definition of heart disease over time.

As increasing age is a natural driver for the incidence of cardiovascular diseases and cardiovascular mortality among the general population, whether the mortality is fully correlated with the death of $\mathrm{BCa}$ remains unclear (11). Patients with BCa have received different treatments, and their cardiac death rates are significantly distinct. For instance, radiotherapy (RT) has been shown to increase the mortality of some particular cancers, while chemotherapy (CT) drugs also have exhibited cardiotoxicity $(12,13)$.

At present, the cardiac-specific mortality of $\mathrm{BCa}$ patients using different treatments has not been analyzed and compared. In view of the possible treatment-related cardiotoxic effects, we aim to analyze the cardiac-specific mortality of a wide range of BCa patients, including 39,500 BCa patients undergoing surgery, RT, or CT. We present the following article in accordance with the STROBE reporting checklist (available at https://dx.doi.org/10.21037/ tau-21-835).

\section{Methods}

\section{Data sources}

The comprehensive demographic and cancer-specific information were obtained from the Surveillance, Epidemiology, and End Results (SEER) database. The cause of death was assigned according to the tenth revision of the International Classification of Diseases (ICD-10). The study was conducted in accordance with the Declaration of Helsinki (as revised in 2013).

\section{Patient selection}

The current analysis was limited to men over the age of 15 who were diagnosed with primary malignant $\mathrm{BCa}$ between 1 January 2000 and 31 December 2012. Patients with unknown treatment, unknown survival outcome, and lack of mortality data were excluded. The follow-up period ended on the 31 December 2015.

\section{Study variables}

Definitions and information regarding variables such as age at diagnosis, year of diagnosis, marital status, and ethnicity, tumor histopathological type, cancer stage, tumor grade, and treatment [radical cystectomy (RC), RT, or CT] was obtained from the SEER database. Patients were stratified by treatment (RC, RT/RT + CT, and CT) age at diagnosis $(<50,50-64,65-74$, or $\geq 75 \mathrm{y})$, and year of diagnosis (year 2000-2005 or 2006-2012).

\section{Statistical analysis}

The composition ratio was used to describe the baseline characteristic distribution, and the chi-squared test was used to evaluate the comparison of composition ratios. A competitive risk model was used to estimate the $\mathrm{BCa}$ specific crude cumulative mortality and heart-specific crude cumulative mortality of $\mathrm{BCa}$ patients, and to plot the crude cumulative mortality curve. The Fine-Gray competitive risk model was used to adjust for the confounding effects of clinical features, and to evaluate the risk of $\mathrm{BCa}$-specific death and heart-specific death in patients undergoing RC, RT, or CT. Cox proportional hazards regression was used to 


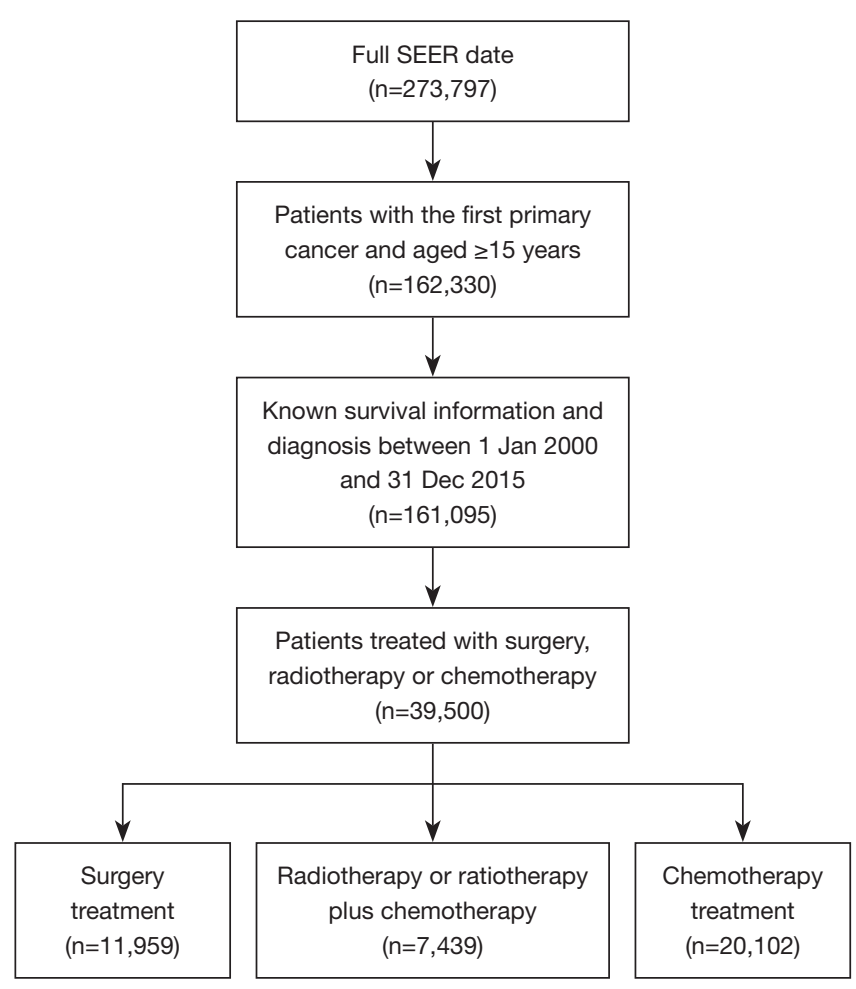

Figure 1 Illustration of the patient selection process. SEER, Surveillance, Epidemiology, and End Results.

evaluate the risk of all-cause mortality. In the groupings of this study, RC included those who received RC alone, RC combined with CT, and RC combined with RT.

To compare mortality between the patients with $\mathrm{BCa}$ and the general population, mortality in those with $\mathrm{BCa}$ was further stratified according to age and stage, and standardized mortality ratios (SMRs) were estimated using the all-cause mortality and heart-specific mortality of the general population. The cause specific SMR was the ratio of the total number of deaths observed to the expected number of deaths for a specific age reference.

The age-specific mortality of the general male population in the United States was obtained from Wide-ranging Online Data for Epidemiologic Research (WONDER), an epidemiological internet-based database maintained by Centers for Disease Control and Prevention (CDC) (14). Statistical analysis was performed and figures were prepared using Stata/MP, version 14.0 and $\mathrm{R}$ software package. $\mathrm{P}<0.05$ (two-sided) was considered to be statistically significant.

\section{Results}

\section{Patients characteristics}

Among the 273,797 BCa cases recorded in SEER database, $162,330(59.3 \%)$ patients were first primary cancer and aged over 15 years old. Of these patients, 161,095 (99.3\%) were diagnosed with $\mathrm{BCa}$ and had full survival data between January 1st 2000 and December 31st 2015. Among these patients, 39,500 (24.5\%) were treated with surgery, RT, or $\mathrm{CT}$ and were eligible for inclusion in this study (Figure 1).

We compared the basic clinical data of the three groups of patients with BCa undergoing RC (30.3\%), CT (50.9\%), or RT or both (18.8\%) (Table 1). The majority of patients were married $(59.8,49.2$, and 61.3 , respectively), white (87.5, 85.2, and 88.2, respectively), and diagnosed between 2006 and 2012 (64.4, 65.5, and 84.8, respectively).

The age distribution was as follows: patients younger than 50 years old contributed the smallest part, while the $50-64,65-74$, and $\geq 75$ years age groups were relatively even. Patients aged $<50,50-64$, and $65-74$ years were more likely to receive RC $(7.9 \%, 36.3 \%$, and $32.5 \%$, respectively), although this was only marginally higher than patients administered CT $(6.4 \%, 30.6 \%$, and $31 \%$, respectively). However, as for men aged $\geq 75$ years, the majority was treated with RT/RT + CT (58.1\%).

Patients with tumor grades III and IV accounted for $73.8 \%$. Of these, RC-treated patients accounted for $39.6 \%$ (grade III) and 38.8\% (grave IV), RT/RT + CT-treated patients accounted for $25 \%$ (grade III) and $21.4 \%$ (grave IV), and CT-treated patients accounted for $35.4 \%$ (grade III), $39.8 \%$ (grave IV). Meanwhile, patients with tumor grade II were mainly administered CT (80.2\%).

\section{Cumulative mortality}

We compared the cumulative mortality in patients with $\mathrm{BCa}$ in terms of three different causes of mortality: tumorspecific death, diseases of the heart, and other causes. Among all patients, the cumulative incidence of BCa was the highest, which was nearly four times the figure for diseases of the heart and twice the figure of other causes in patients who survived 50-200 months after diagnosis. Specifically, for patients with a survival period of less than 50 months, tumor-specific death exhibited a rapidly increasing trend, which subsequently flatlined. However, compared to tumor-specific mortality, However, the rates heart-specific 
Table 1 Basic characteristics at diagnosis of patients with BCa in the RC, RT/RT + CT, or CT treatment groups

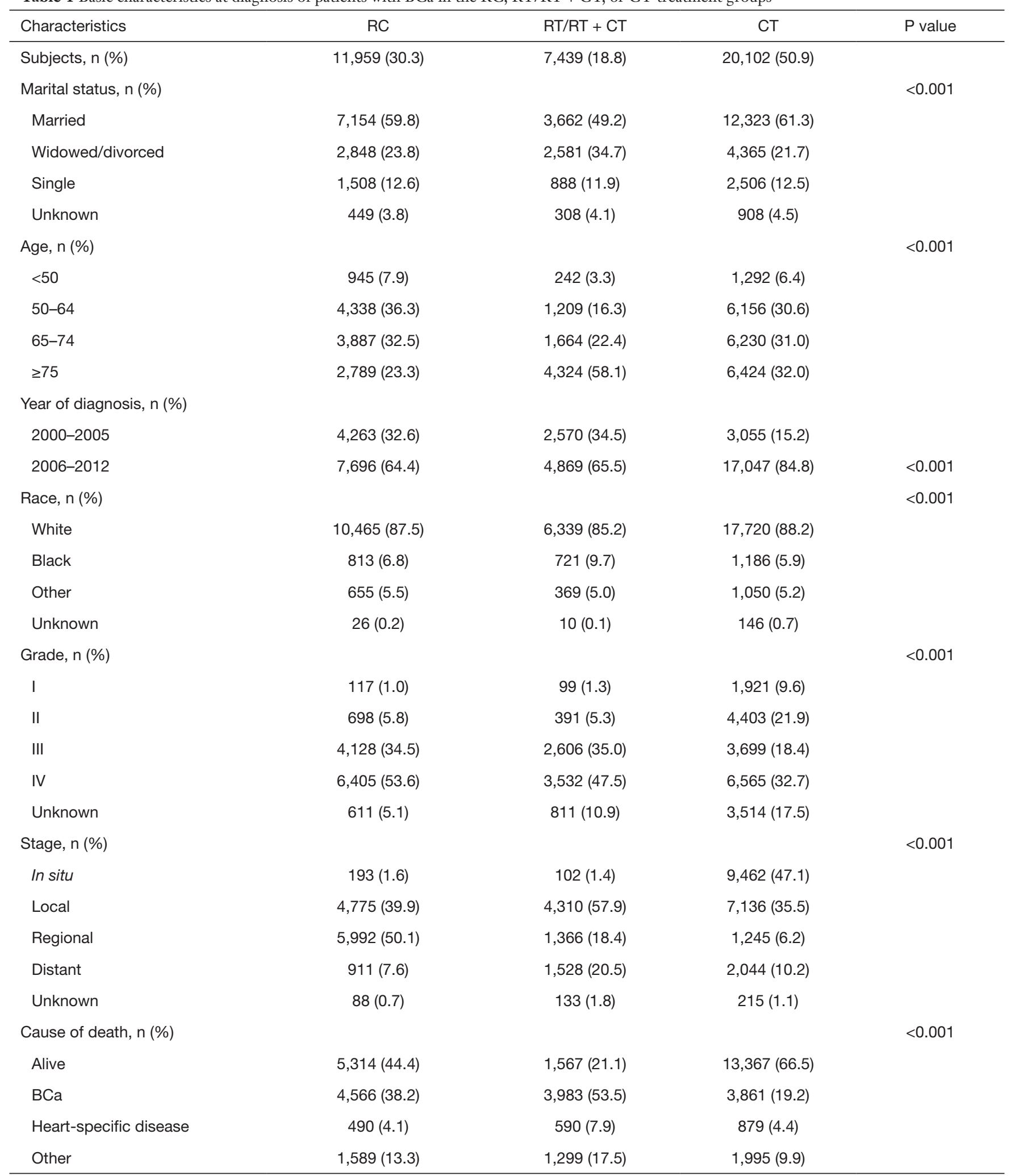

$\mathrm{BCa}$, bladder cancer; $\mathrm{RC}$, radical cystectomy; RT, radiotherapy; CT, chemotherapy. 

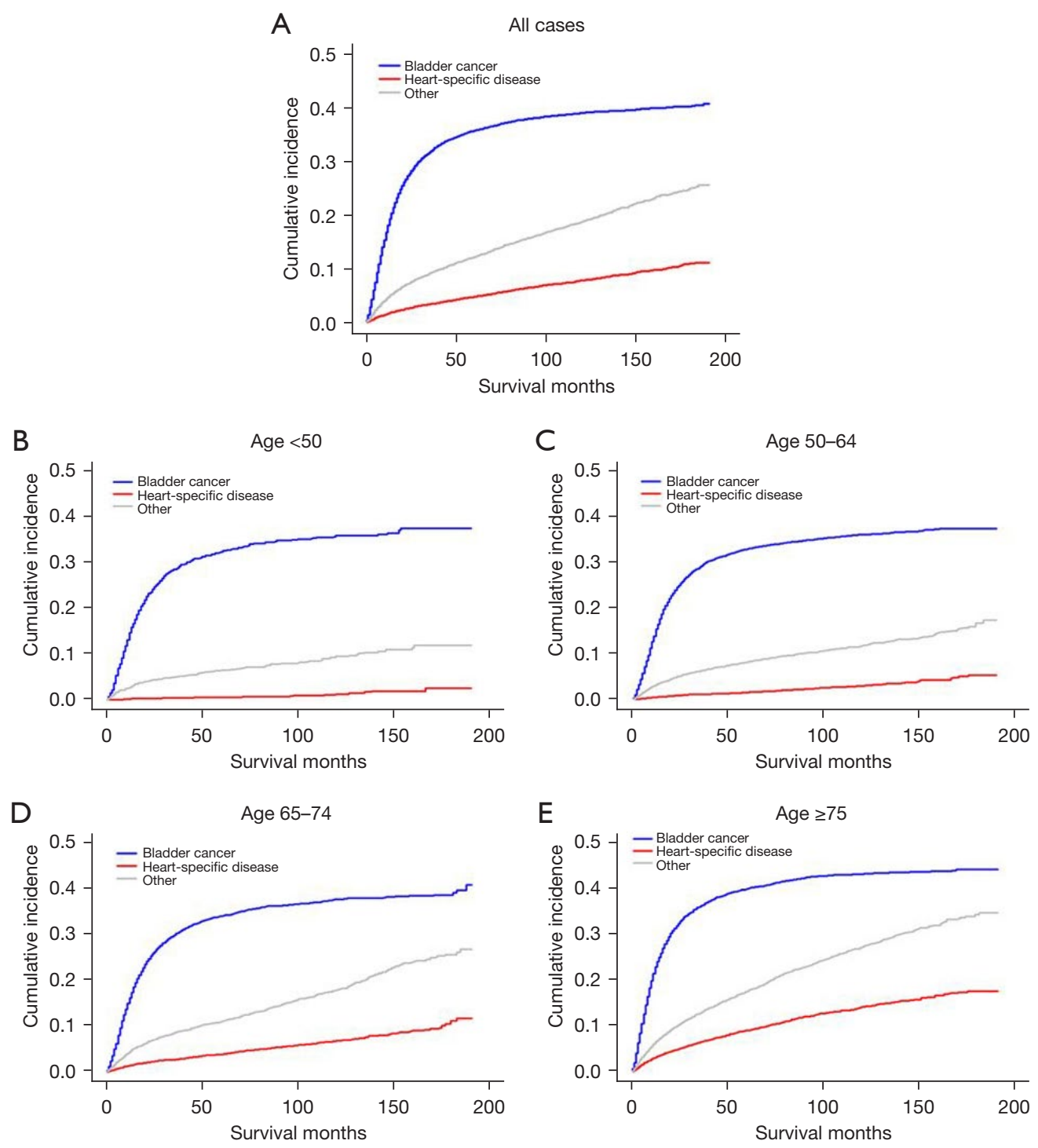

Figure 2 Multivariable adjusted cumulative incidence function curve of patients with BCa. (A) Cause-specific mortality of the overall BCa population. (B-E) Heart-specific and BCa mortality rates by age at diagnosis: (B) <50 y; (C) 50-64 y; (D) 65-74 y; and (E) $\geq 75$ y. BCa, bladder cancer.

mortality and other causes exhibited a tendency to increase stably. This suggests that there will be a continuous rise in the number in patients who live longer (Figure $2 A$ ).

We also explored the data of patients by the different age groups, 50-64, 65-74, and $\geq 75$ years, and found that the composition and changing trend of the causes of death in all age groups were similar to that of the entire population. Nevertheless, for older patients (65-74 and $\geq 75$ years), the number of heart-specific deaths was significantly increased, especially in patients with a relatively long survival period (longer than 50 months) (Figure 2B-2E).

The lowest mortality belonged to patients that received surgery and any of the other treatment strategies after $\mathrm{BCa}$ diagnosis (Figure $3 A, 3 B$ ). The percentage of those who underwent RT/RT + CT outnumbered that of patients who chose CT only (Figure $3 A$ ). By further analyzing the various treatment methods separately, two treatment methods that included RT exhibited the top two mortality rates, followed by CT, with surgery still maintaining a low mortality rate (Figure 3C). 

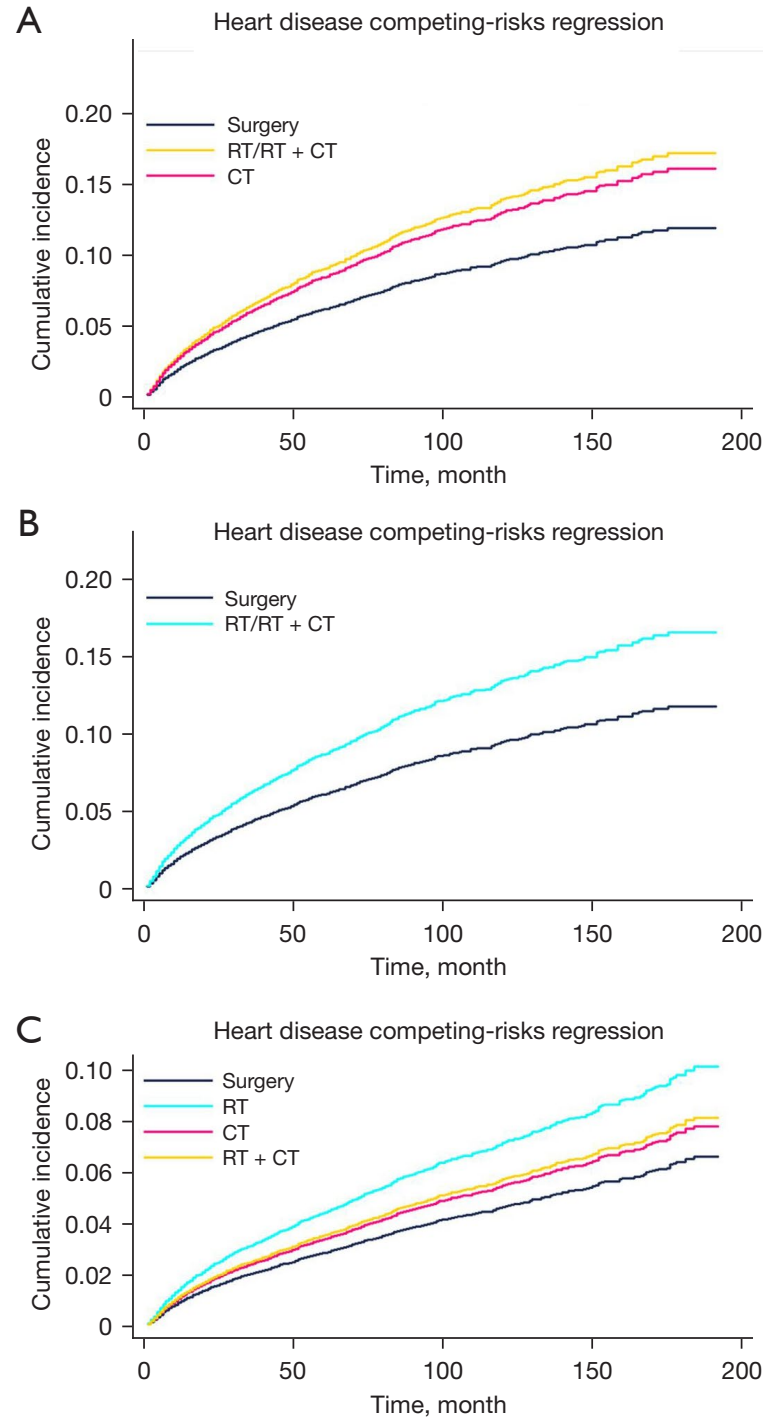

Figure 3 The heart-specific competing-risks regression of patients with $\mathrm{BCa}$ undergoing $\mathrm{RC}, \mathrm{RT}$ or CT. BCa patients were grouped as follows: (A) RC, RT/RT + CT, and CT; (B) RC and RT/RT + CT/CT; (C) RC, RT, CT, and RT + CT. BCa, bladder cancer; RC, radical cystectomy; RT, radiotherapy; CT, chemotherapy.

\section{Mortality compared with the general population}

The heart-specific and all-cause mortality rates of patients with BCa undergoing RC, RT, or CT were standardized by age and cancer stage relative to the general population (Table 2). Overall, the heart-specific and all-cause mortality rates of patients of any age groups treated with the three aforementioned strategies were higher than those of general population. The all-cause mortality of patients was generally five times (or higher) than that of heart-specific mortality. Along with increasing age, the all-cause mortality rate of $\mathrm{BCa}$ patients exhibited a continuous downward trend. However, the heart-specific mortality of patients who received RC or CT fluctuated around SMR 2, while that of patients who underwent RT showed an initially increasing trend that subsequently decreased, reaching a peak in the 65-70 age group (SMR $=7.53$; 95\% CI: 5.46-10.39).

We also grouped the patients according to tumor stages, and found that the heart-specific mortality of patients with carcinoma in situ treated by RC and CT exceeded their all-cause mortality, while that of other stages did not. The all-cause mortality rate of patients with distant tumor metastasis was the highest among all patients, regardless of treatment (RC: SMR $=32.17$, 95\% CI: 29.78-34.75; RT/ RT + CT: SMR $=45.25,95 \%$ CI: 42.67-47.99; CT: SMR $=38.74,95 \%$ CI: $36.83-40.74)$.

\section{Relative risk model for specific causes}

We established a Fine-Gray model of competitive risk to explore the internal logic of the prognostic factors of heart disease or BCa mortality in patients receiving RC, RT, or CT (Table 3). Taking 5 years as a time period, the risk of heart-specific death increased from 2.32 (50-64 years) to 10.72 ( $\geq 75$ years) with the increase in the patient's age. This trend was consistent with that of the risk of tumor-specific death in patients, where patients over 75 years of age had a higher risk of dying from tumors [sub-distribution hazard ratio $(\mathrm{SHR})=1.39 ; 95 \%$ CI: $1.28-1.50]$. Patients with $\mathrm{BCa}$ diagnosed between 2006 and 2012 (SHR $=0.59$; 95\% CI: 0.54-0.65) had a significantly lower risk of heart-specific death than those diagnosed in 2000-2005. Compared with other tumor stages, patients with distant metastasis had the lowest risk of heart-specific death $(\mathrm{SHR}=0.37$; $95 \% \mathrm{CI}$ : 0.29-0.47), but their risk of tumor-specific death, which gradually increased with tumor progression, was several times higher than that of the other stages ( $\mathrm{SHR}=48.79$; 95\% CI: 41.91-56.79).

The risks of heart-specific $(\mathrm{SHR}=1.38 ; 95 \% \mathrm{CI}$ : $1.22-1.57$ ) and tumor-specific deaths (SHR $=1.68 ; 95 \%$ CI: $1.60-1.77)$ in patients who received RT were higher than those of patients who underwent CT. The stratified study based on different ages showed that the risk of heartspecific death in patients over 65 years of age who received RT treatment was higher than that of patients who received other treatments (Table 4). Similarly, patients over 75 years of age who underwent RT had a relatively higher risk of 
Table 2 The heart-specific and all-cause mortality rates of patients with BCa undergoing RC, RT, or CT were standardized by age and cancer stage relative to the general population

\begin{tabular}{|c|c|c|c|c|c|c|}
\hline Group & \multicolumn{3}{|c|}{ SMR heart-specific $(95 \% \mathrm{Cl})$} & \multicolumn{3}{|c|}{ SMR overall (95\% Cl) } \\
\hline \multicolumn{7}{|l|}{ Age } \\
\hline $50-54$ & $2.72(1.41,5.22)$ & $4.49(1.12,17.97)$ & $1.27(0.53,3.06)$ & $24.42(22.13,26.93)$ & $76.56(65.81,89.06)$ & $14.04(12.46,15.81)$ \\
\hline $55-59$ & $2.92(1.94,4.39)$ & $2.54(0.82,7.88)$ & $1.76(1.09,2.83)$ & $16.55(15.3,17.91)$ & $49.9(44.38,56.1)$ & $10.72(9.82,11.71)$ \\
\hline $65-69$ & $2.75(2.16,3.5)$ & $7.53(5.46,10.39)$ & $1.88(1.46,2.41)$ & $9.21(8.66,9.79)$ & $21.72(19.88,23.72)$ & $5.28(4.92,5.66)$ \\
\hline $70-74$ & $2.18(1.74,2.73)$ & $6.44(4.98,8.33)$ & $1.79(1.45,2.21)$ & $6.62(6.23,7.03)$ & $15.57(14.42,16.82)$ & $3.93(3.67,4.19)$ \\
\hline $75-79$ & $1.91(1.56,2.33)$ & $4.33(3.49,5.38)$ & $2(1.71,2.35)$ & $4.54(4.27,4.84)$ & $10.86(10.18,11.58)$ & $2.93(2.76,3.12)$ \\
\hline $80-85$ & $1.63(1.35,1.98)$ & $3.44(2.92,4.04)$ & $1.54(1.33,1.79)$ & $3.35(3.13,3.58)$ & $6.64(6.27,7.03)$ & $2.25(2.12,2.4)$ \\
\hline Local & $1.65(1.42,1.92)$ & $4.41(3.9,4.99)$ & $2.14(1.89,2.43)$ & $3.31(3.14,3.48)$ & $8.26(7.91,8.63)$ & $3.9(3.73,4.08)$ \\
\hline Regional & $2.51(2.19,2.88)$ & $3.5(2.63,4.64)$ & $3.12(2.25,4.33)$ & $9.71(9.4,10.03)$ & $13.4(12.5,14.36)$ & $14.9(13.88,16)$ \\
\hline Distant & $3.56(2.18,5.8)$ & $5.53(3.89,7.86)$ & $3.4(2.38,4.86)$ & $32.17(29.78,34.75)$ & $45.25(42.67,47.99)$ & $38.74(36.83,40.74)$ \\
\hline Unknown & $1.19(0.3,4.75)$ & $3.5(1.46,8.41)$ & $1.85(0.83,4.11)$ & $7.14(5.46,9.35)$ & $12.33(9.85,15.44)$ & $7.33(6.05,8.88)$ \\
\hline Total & $2.08(1.89,2.29)$ & $4.27(3.84,4.75)$ & $1.79(1.64,1.94)$ & $6.68(6.51,6.85)$ & $11.75(11.39,12.12)$ & $3.86(3.76,3.97)$ \\
\hline
\end{tabular}

$\mathrm{BCa}$, bladder cancer; RC, radical cystectomy; RT, radiotherapy; $\mathrm{CT}$, chemotherapy; SMR, standardized mortality ratio; Cl, confidence interval.

tumor-specific death (SHR =1.62; 95\% CI: $1.50-1.75)$.

\section{Discussion}

In this study, which was based on mega data and a large sample of patients, we analyzed the long-term heart-specific mortality of BCa patients. Overall, the results of the study showed that the heart-specific mortality rate in BCa patients diagnosed in recent years was significantly lower than that of patients diagnosed in earlier years. This may be related to the improvement of the precision of surgery, RT, and $\mathrm{CT}$ as well as the quality of comprehensive management of tumors, which has fewer complications and side effects. At the same time, the treatment of cardiovascular diseases and the ability to respond to cardiovascular events have been improved, which has also effectively reduced heart-specific deaths.

For BCa, awareness of hematuria as the major presenting symptom is paramount, and early diagnosis with individualized treatment and follow-up is key to a successful outcome. In terms of BCa surgical treatment, with the increased popularity of robotic surgery in recent years, numerous studies have compared the perioperative results and long-term progression-free survival of robotassisted RC (RARC) and RC $(15,16)$. The results have demonstrated that RARC is technically feasible, provides the same perioperative and oncological results compared with RC, and has the potential advantage of being more refined (17). Increased development may further shorten the operation time and reduce the increase in the patient's heart load caused by anesthesia (18). However, RARC also has high costs. The development of the technique can bring about better treatment.

A general analysis has shown that older patients have a higher potential for death due to cardiac-specific causes. At present, the influence of age on the risk of cardiovascular disease cannot be ruled out. The significant enhancement of medical oncology treatment, especially the clinical application of immunotherapy, enables patients to achieve a longer survival period, combined with the risk factors of degenerative changes in aging patients. Therefore, we further stratified our assessment based on age and tried to 
Table 3 All-cause, heart-related, and bladder-related mortality of patients by demographic and clinical characteristics at diagnosis

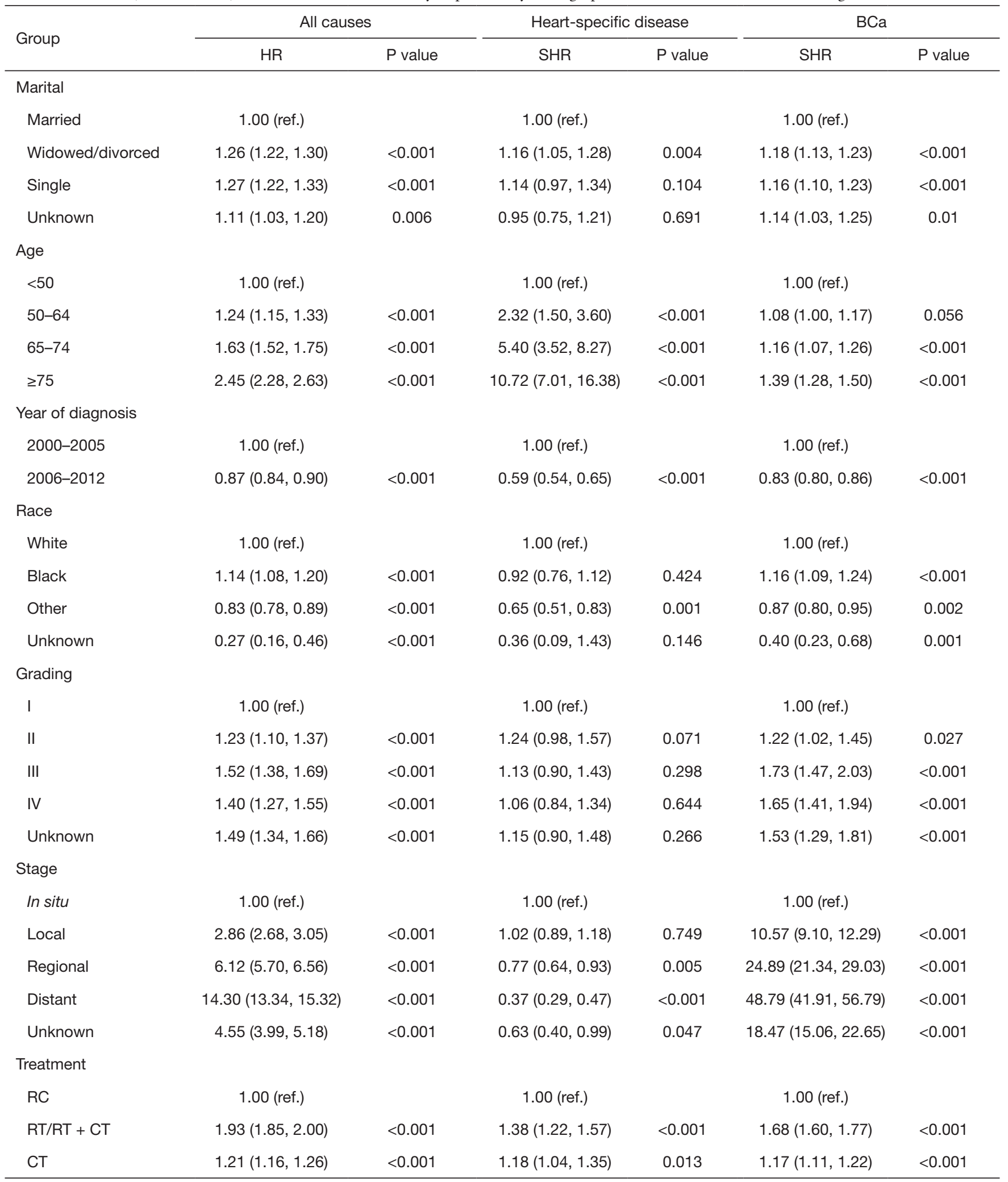

$\mathrm{BCa}$, bladder cancer; HR, hazard ratio; SHR, sub-distribution hazard ratio; RC, radical cystectomy; RT, radiotherapy; CT, chemotherapy. 
Table 4 All-cause, heart-related, and bladder-related mortality in patients with BCa who underwent RC, RT/RT + CT, or CT at diagnosis, analyzed by age and treatment subgroup

\begin{tabular}{|c|c|c|c|c|c|c|c|}
\hline Age & Variables & \multicolumn{2}{|c|}{ All causes } & \multicolumn{2}{|c|}{ Heart-specific disease } & \multicolumn{2}{|c|}{$\mathrm{BCa}$} \\
\hline \multirow[t]{3}{*}{$<50$} & Treatment & & & & & & \\
\hline & $\mathrm{RC}$ & & & & & & \\
\hline & $\mathrm{RT} / \mathrm{RT}+\mathrm{CT}$ & $2.15(1.78,2.60)$ & $<0.001$ & $1.62(0.52,5.12)$ & 0.407 & $1.89(1.50,2.39)$ & $<0.001$ \\
\hline \multirow[t]{4}{*}{$50-64$} & Treatment & & & & & & \\
\hline & $\mathrm{RC}$ & & & & & & \\
\hline & $\mathrm{RT} / \mathrm{RT}+\mathrm{CT}$ & $1.84(1.69,2.00)$ & $<0.001$ & $1.29(0.83,2.00)$ & 0.261 & $1.67(1.51,1.86)$ & $<0.001$ \\
\hline & $\mathrm{CT}$ & $1.27(1.18,1.37)$ & $<0.001$ & $1.00(0.69,1.46)$ & 0.981 & $1.26(1.16,1.38)$ & $<0.001$ \\
\hline \multirow{2}{*}{$65-74$} & $\mathrm{RT} / \mathrm{RT}+\mathrm{CT}$ & $1.90(1.76,2.05)$ & $<0.001$ & $1.59(1.25,2.02)$ & $<0.001$ & $1.57(1.43,1.73)$ & $<0.001$ \\
\hline & CT & $1.17(1.09,1.26)$ & $<0.001$ & $1.00(0.79,1.27)$ & 0.995 & $1.20(1.09,1.31)$ & $<0.001$ \\
\hline \multirow[t]{4}{*}{$\geq 75$} & Treatment & & & & & & \\
\hline & $\mathrm{RC}$ & & & & & & \\
\hline & $\mathrm{RT} / \mathrm{RT}+\mathrm{CT}$ & $1.98(1.86,2.10)$ & $<0.001$ & $1.49(1.25,1.77)$ & $<0.001$ & $1.62(1.50,1.75)$ & $<0.001$ \\
\hline & CT & $1.17(1.10,1.26)$ & $<0.001$ & $1.38(1.15,1.66)$ & 0.001 & $1.01(0.93,1.11)$ & 0.754 \\
\hline
\end{tabular}

$\mathrm{BCa}$, bladder cancer; RC, radical cystectomy; RT, radiotherapy; CT, chemotherapy; HR, hazard ratio; SHR, sub-distribution hazard ratio.

eliminate the problems of aging and degeneration caused by age itself. For patients over 65 years who received RT, the heart-specific mortality rate was significantly higher than that of the RC and CT groups. Meanwhile, the heartspecific mortality risk of the other age groups who received any of the three treatments was not significant different to that of the general population.

Radiation-induced heart disease (RIHD) is a unifying term used to describe all cardiac complications related to RT. The pathophysiological process of RIHD mainly includes DNA damage caused by radiation and oxidative stress, which ultimately leads to endothelial damage (19). RT may accelerate the process of atherosclerosis, venous thromboembolism, early coronary artery disease, valvular heart disease, and heart failure (20). In recent years, the rapid development of cytotoxic CT, molecular targeted inhibitors, and immune checkpoint therapy (target cytotoxic $\mathrm{T}$ lymphocyte antigen 4 , programmed cell death-1, and PD-ligand 1) are mostly directed at signal transduction pathways and immune regulation. These methods have limited long-term cardiovascular toxicity data due to their short application life $(21,22)$.

Cardiotoxicity is a potential complication of various tumor treatments. The ESMO clinical practice guidelines are the result of a multidisciplinary review of cardiology and oncology. Its ultimate goal is to provide strict standard recommendations for the prevention, evaluation, monitoring and management of cardiotoxicity risks during anti-cancer treatment (23). In principle, the risk-benefit ratio of drugs must be explained according to the nature and severity of the disease.

The establishment of the prognostic risk model suggested that risk stratification and pre-treatment screening will be included in the diagnosis and treatment of BCa patients, which can detect and predict the effects that can reduce the risk of cardiac-specific death and benefit patients. This analytical model showed that patients in different tumor stages have different courses of progression, and those with distant metastases have a low risk of heart-specific death. This result needs to be analyzed with survival time and 
other factors. Analyze the prognostic risk of patients using a single factor is unscientific.

At present, there is a lack of guidelines for the management of RT-related cardiac complications in $\mathrm{BCa}$ patients.

However, the model in this paper has certain limitations because it does not consider important information, such as RT type, strategy, dose, CT plan, time, etc. Also, some patients who died of unknown causes were included, and the specific types of cardiac-specific deaths were not discussed. The general information in the SEER database was not sufficient, and we cannot rule out whether it had a specific effect.

\section{Acknowledgments}

We would like to thank the staff members of the National Cancer Institute and their colleagues across the United States and at Information Management Services, Inc., who have been involved with the Surveillance, Epidemiology, and End Results (SEER) Program.

Funding: This work was supported in part by grants from the Shanghai Science Committee Foundation (\#19411967700), the Shanghai Municipal Health Commission Fund (202040179), and the National Natural Science Foundation of China (82101838).

\section{Footnote}

Reporting Checklist: The authors have completed the STROBE reporting checklist. Available at https://dx.doi. org/10.21037/tau-21-835

Conflicts of Interest: All authors have completed the ICMJE uniform disclosure form (available at https://dx.doi. org/10.21037/tau-21-835). The authors have no conflicts of interest to declare.

Ethical Statement: The authors are accountable for all aspects of the work in ensuring that questions related to the accuracy or integrity of any part of the work are appropriately investigated and resolved. The study was conducted in accordance with the Declaration of Helsinki (as revised in 2013).

Open Access Statement: This is an Open Access article distributed in accordance with the Creative Commons Attribution-NonCommercial-NoDerivs 4.0 International
License (CC BY-NC-ND 4.0), which permits the noncommercial replication and distribution of the article with the strict proviso that no changes or edits are made and the original work is properly cited (including links to both the formal publication through the relevant DOI and the license). See: https://creativecommons.org/licenses/by-nc-nd/4.0/.

\section{References}

1. Grayson M. Bladder cancer. Nature 2017;551:S33.

2. Chavan S, Bray F, Lortet-Tieulent J, et al. International variations in bladder cancer incidence and mortality. Eur Urol 2014;66:59-73.

3. Burger M, Catto JW, Dalbagni G, et al. Epidemiology and risk factors of urothelial bladder cancer. Eur Urol 2013;63:234-41.

4. Freedman ND, Silverman DT, Hollenbeck AR, et al. Association between smoking and risk of bladder cancer among men and women. JAMA 2011;306:737-45.

5. Compérat $\mathrm{E}$, Larré $\mathrm{S}$, Roupret $\mathrm{M}$, et al. Clinicopathological characteristics of urothelial bladder cancer in patients less than 40 years old. Virchows Arch 2015;466:589-94.

6. Lenis AT, Lec PM, Chamie K, et al. Bladder cancer: a review. JAMA 2020;324:1980-91.

7. Hanna TP, King WD, Thibodeau S, et al. Mortality due to cancer treatment delay: systematic review and metaanalysis. BMJ 2020;371:m4087.

8. Hajiran A, Azizi M, Aydin AM, et al. Pathological and survival outcomes associated with variant histology bladder cancers managed by cystectomy with or without neoadjuvant chemotherapy. J Urol 2021;205:100-8.

9. Abdel-Rahman O. Risk of cardiac death among cancer survivors in the United States: a SEER database analysis. Expert Rev Anticancer Ther 2017;17:873-8.

10. Roth GA, Mensah GA, Johnson CO, et al. Global burden of cardiovascular diseases and risk factors, 1990-2019: update from the GBD 2019 study. J Am Coll Cardiol 2020;76:2982-3021.

11. Sharabi AB, Lim M, DeWeese TL, et al. Radiation and checkpoint blockade immunotherapy: radiosensitisation and potential mechanisms of synergy. Lancet Oncol 2015;16:e498-509.

12. Ali A, Song YP, Mehta S, et al. Palliative radiation therapy in bladder cancer-importance of patient selection: a retrospective multicenter study. Int J Radiat Oncol Biol Phys 2019;105:389-93.

13. Bajaj A, Martin B, Bhasin R, et al. The impact of 
academic facility type and case volume on survival in patients undergoing curative radiation therapy for muscle-invasive bladder cancer. Int J Radiat Oncol Biol Phys 2018;100:851-7.

14. Zhang E, He X, Yin D, et al. Increased expression of long noncoding RNA TUG1 predicts a poor prognosis of gastric cancer and regulates cell proliferation by epigenetically silencing of p57. Cell Death Dis 2016;7:e2109.

15. Tyritzis SI, Gaya JM, Wallestedt-Lantz A, et al. Current role of robotic bladder cancer surgery. Minerva Urol Nefrol 2019;71:301-8.

16. Sohn W, Lee HJ, Ahlering TE. Robotic surgery: review of prostate and bladder cancer. Cancer J 2013;19:133-9.

17. Parekh DJ, Reis IM, Castle EP, et al. Robot-assisted radical cystectomy versus open radical cystectomy in patients with bladder cancer (RAZOR): an open-label, randomised, phase 3, non-inferiority trial. Lancet 2018;391:2525-36.

18. Vatner SF. Effects of anesthesia on cardiovascular control mechanisms. Environ Health Perspect 1978;26:193-206.

19. Zamorano JL, Lancellotti P, Rodriguez Muñoz D, et

Cite this article as: Yang F, Li C, Guo Y, Yu Y, Mao S, Wang R, Zhang W, Zhang A, Yao X. Effects of radical cystectomy, radiotherapy, and chemotherapy on the risk of long-term heartspecific death in bladder cancer patients. Transl Androl Urol 2021;10(10):3826-3836. doi: 10.21037/tau-21-835 al. 2016 ESC Position Paper on cancer treatments and cardiovascular toxicity developed under the auspices of the ESC Committee for Practice Guidelines: The Task Force for cancer treatments and cardiovascular toxicity of the European Society of Cardiology (ESC). Eur Heart J 2016;37:2768-801.

20. Raghunathan D, Khilji MI, Hassan SA, et al. Radiationinduced cardiovascular disease. Curr Atheroscler Rep 2017;19:22.

21. Totzeck M, Schuler M, Stuschke M, et al. Cardio-oncology - strategies for management of cancer-therapy related cardiovascular disease. Int J Cardiol 2019;280:163-75.

22. Zhou YW, Zhu YJ, Wang MN, et al. Immune checkpoint inhibitor-associated cardiotoxicity: current understanding on its mechanism, diagnosis and management. Front Pharmacol 2019;10:1350.

23. Curigliano G, Cardinale D, Suter T, et al. Cardiovascular toxicity induced by chemotherapy, targeted agents and radiotherapy: ESMO Clinical Practice Guidelines. Ann Oncol 2012;23 Suppl 7:vii155-66. 